\title{
P04-07. Guiding the immune system through complex maturation pathways: a novel multi-immunogen approach for elicitation of broadly neutralizing antibodies X Xiao*1, Y Feng1, W Chen ${ }^{1}$, N Longo ${ }^{2}$ and DS Dimitrov ${ }^{1}$
}

Address: ${ }^{1}$ CCRNP, NCI, NIH, Frederick, USA and ${ }^{2}$ National Insitute of Arthritis and Musculoskeletal and Skin Disease, Bethesda, MD, USA

* Corresponding author

from AIDS Vaccine 2009

Paris, France. 19-22 October 2009

Published: 22 October 2009

Retrovirology 2009, 6(Suppl 3):P35 doi:10.1 I86/1742-4690-6-S3-P35

This abstract is available from: http://www.retrovirology.com/content/6/S3/P35

(c) 2009 Xiao et al; licensee BioMed Central Ltd.

\section{Background}

Several human monoclonal antibodies (hmAbs) including Fab X5, b12, 2G12 and 2F5 exhibit relatively potent and broad HIV-1 neutralizing activity. These antibodies are highly divergent from the closest corresponding germline antibodies. We have hypothesized that the high degree of specific somatic hypermutations may preclude binding of the HIV-1 envelope glycoprotein (Env) to their corresponding germline antibodies, and that identifying antibodies that are intermediates in the pathways to maturation could help design novel vaccine immunogens. Such immunogens may not be based on the Env but help guide the immune system through the long complex pathways to maturation.

\section{Methods}

We have designed germline-like antibodies where several different CDR3s of the heavy chain were used including one which is identical to that of the mature antibody.

\section{Results}

The germline-like X5 bound with high affinity to all tested Envs and neutralized a panel of HIV-1 isolates from different clades. In contrast, none of the other germline-like antibodies (b12, 2G12 and 2F5) including antibodies in multivalent formats bound to any of the Envs and did not neutralize any of the HIV-1 isolate tested although the corresponding mature antibodies did with high level of activity. Because b12 is best characterized in terms of structure/ function relationships we selected it for characterization of possible maturation pathways. We identified a panel of possible b1 2 intermediate antibodies and several antigens not related to the Env that bind to some of these antibodies.

\section{Conclusion}

These antigens together with Envs could be used as a conceptually novel type of candidate vaccines based on two or more immunogens that help guiding the immune system through the complex maturation pathways for elicitation of antibodies that are similar or identical to antibodies with known properties. 